\title{
Monte Carlo Simulations of Coupled Diffusion and \\ Surface Reactions during the Aqueous Corrosion of \\ Borosilicate Glasses
}

\author{
Sebastien Kerisit ${ }^{a^{*}}$, Eric M. Pierce ${ }^{b}$, and Joseph V. Ryan ${ }^{c}$ \\ ${ }^{a}$ Fundamental and Computational Sciences Directorate, Pacific Northwest National Laboratory, Richland, WA \\ 99352, USA \\ ${ }^{\mathrm{b}}$ Energy and Environmental Sciences Directorate, Oak Ridge National Laboratory, Oak Ridge, TN 37831, USA \\ ${ }^{c}$ Energy and Environment Directorate, Pacific Northwest National Laboratory, Richland, WA 99352, USA \\ "Corresponding Author: S. Kerisit - sebastien.kerisit@pnnl.gov; +1 5093716382.
}

\begin{abstract}
Borosilicate nuclear waste glasses develop complex altered layers as a result of coupled processes such as hydrolysis of network species, condensation of Si species, and diffusion. However, diffusion has often been overlooked in Monte Carlo models of the aqueous corrosion of borosilicate glasses. Therefore, three different models for dissolved Si diffusion in the altered layer were implemented in a Monte Carlo model and evaluated for glasses in the compositional range $(75-x) \mathrm{mol} \% \mathrm{SiO}_{2}(12.5+x / 2) \mathrm{mol} \% \mathrm{~B}_{2} \mathrm{O}_{3}$ and $(12.5+x / 2)$ mol $\% \mathrm{Na}_{2} \mathrm{O}$, where $0 \leq x \leq 20 \%$, and corroded in static conditions at a surface-area-to-volume ratio of $1000 \mathrm{~m}^{-1}$. The three models considered instantaneous homogenization (M1), linear concentration gradients (M2), and concentration profiles determined by solving Fick's $2^{\text {nd }}$ law using a finite difference method (M3). Model M3 revealed that concentration profiles in the altered layer are not linear and show changes in shape and magnitude as corrosion progresses, unlike those assumed in model M2. Furthermore, model M3 showed that, for borosilicate glasses with a high forward dissolution rate compared to the diffusion rate, the gradual polymerization and densification of the altered layer
\end{abstract}


is significantly delayed compared to models M1 and M2. Models M1 and M2 were found to be appropriate models only for glasses with high release rates such as simple borosilicate glasses with low $\mathrm{ZrO}_{2}$ content.

Keywords: Monte Carlo; borosilicate glasses; diffusion; alteration layer; kinetics.

\section{Introduction}

Disposition of radioactive waste generated by the nuclear fuel cycle and nuclear weapons production during the Cold War era is one of the most pressing environmental challenges facing the United States and the international community [1,2]. The preferred approach for long-term storage of nuclear waste involves immobilization via solidification in glass (e.g. borosilicates) and disposal in subsurface [3,4]. Consequently, there is a critical need for predicting the longterm behavior of glasses in disposal conditions, specifically the rates of radionuclide release, which, in turn, requires a comprehensive understanding of glass-water reactions.

Our understanding of the aqueous corrosion of borosilicate nuclear waste glasses has grown considerably over the last few decades [5]. Borosilicate glasses are known to develop an altered layer in non-dilute conditions as a result of several coupled processes, namely, ion exchange, hydrolysis of network species, condensation of moderately soluble species such as silica, and precipitation of secondary crystalline phases. The altered layer begins at the interface with the pristine glass and ends at the interface with the aqueous solution. It consists mostly of an amorphous hydrated surface layer with crystalline alteration products generally found at the interface with the aqueous solution. Although much progress has been made to date, understanding the complex physical and chemical properties of the altered layer remains a 
formidable challenge and the main obstacle for developing models that can accurately predict the long-term rates of radionuclide release.

Significant insights into the structure and properties of the altered layer have been gained from experiment [6-10]; but experimental measurements have also been supplemented appreciably by theoretical and computational modeling studies [11-15]. In particular, Monte Carlo (MC) models have shown success in simulating the interplay between the microscopic processes that underlie macroscopic glass corrosion behavior. Notably, early MC models by Aertsens and co-workers [13, 16-19] showed that, in static corrosion conditions, the silica saturation concentration changes with time due to restructuring of the altered layer, which prompted a re-formulation of the linear glass dissolution rate law put forward by Grambow [20, 21]. Later MC simulations by Devreux and co-workers [11, 22-28] emphasized the formation of a dense layer in the outer region of the altered layer that can eventually block the aqueous glass corrosion. This finding explained the unexpected experimental observation that fast-dissolving glasses only show limited extent of corrosion [10, 29]. Recently, Kerisit and co-workers [12, 3032] developed MC models that could account for borosilicate glass dissolution in flow-through conditions, the influence of aluminum on the rates and mechanisms of dissolution, and the formation of secondary phases.

Although success has been achieved for some glass compositions, not all known glass corrosion behaviors can be reproduced by existing Monte Carlo models. One critical process often overlooked in current MC models is diffusion of dissolved Si species through the altered layers. An early MC model of corrosion in static conditions by Aertsens and co-workers did include diffusion of dissolved Si species via hopping to nearest-neighbor sites on the MC lattice [18]. However, the computational expense associated with this approach and the computing 
power available at that time imposed limits on the system size and compositional range that could be studied. Diffusion was not explicitly considered in the MC simulations of Devreux and co-workers; with the exception of one study by Ledieu et al. [27], which attempted to address this issue, albeit with a simple concentration gradient model and for a single glass composition.

Therefore, this work aims to provide a systematic study of the effects of diffusion on the structure and dynamics of the altered layer. Several treatments of diffusion are implemented and tested using sodium borosilicate glasses as a model system. Results are compared to the instantaneous homogenization scenario as well as the simple concentration gradient model of Ledieu et al. [27]. In addition, the effects of glass composition are investigated.

\section{Computational Methods}

All the calculations presented in this work were performed with a Monte Carlo computer program developed by Kerisit and co-workers, as described in previous publications [12, 30-32]. The Monte Carlo model follows the glass dissolution model of Devreux, Barboux and coworkers [11, 24-26].

In the MC model, network-former cations $\mathrm{B}$ and $\mathrm{Si}$ are placed on a three-dimensional (3D) cubic lattice in which each lattice site represents one network-former cation and its first oxygen coordination shell, and two connections are removed so that each site is in four-fold coordination. The network-modifier cation $\mathrm{Na}$ is placed in interstitial positions. When a networkformer cation dissolves, its site is considered to be replaced by water. To obtain a given surfacearea-to-volume ratio, the contacting (bulk) aqueous solution is represented by a fixed number of water sites. 
The simulated glasses are in the compositional range $(75-x) \mathrm{mol} \% \mathrm{SiO}_{2}(12.5+x / 2) \mathrm{mol} \%$ $\mathrm{B}_{2} \mathrm{O}_{3}$ and $(12.5+x / 2) \operatorname{mol} \% \mathrm{Na}_{2} \mathrm{O}$, where $0 \leq x \leq 20 \%$. The lower limit of the $\mathrm{SiO}_{2}$ mole percent (55\%) was chosen to be above the silicon percolation threshold (44\%) [30] and the upper limit was chosen to be above that of the combined mole percent of insoluble and sparingly soluble oxides in typical nuclear waste glasses $[33,34]$. Because the mole fraction of $\mathrm{Na}_{2} \mathrm{O}$ is equal to that of $\mathrm{B}_{2} \mathrm{O}_{3}$, all the $\mathrm{B}$ ions are considered to be in tetrahedral coordination and to be chargecompensated by $\mathrm{Na}$ ions in the simulated glasses.

The three elements considered in this work exhibit different dissolution/condensation behaviors. Boron is considered to dissolve instantaneously as soon as it is in contact with the bulk aqueous solution and to be fully soluble, i.e. it cannot condense back on the glass surface. Dissolution probabilities $w_{1}, w_{2}$, and $w_{3}$ are used for Si sites with one, two, or three connections to nearest-neighbor Si sites. In an effort to reduce the total number of model parameters, the ratios $w_{1} / w_{2}$ and $w_{2} / w_{3}$ are set to be equal and this ratio is designated as $\delta$. Dissolved Si can condense at surface sites with the probability $w_{r}=w_{c-\mathrm{Si}} C_{\mathrm{Si}}$, where $w_{c-\mathrm{Si}}$ is the $\mathrm{Si}$ condensation probability and $C_{\mathrm{Si}}$ is the silicon concentration in the aqueous solution (in atoms per aqueous site). $\mathrm{Na}$ ions are considered to dissolve with the $\mathrm{B}$ ions they are charge-compensating and are not allowed to condense at the glass surface. Therefore, three parameters are required to model the aqueous corrosion of borosilicate glasses, namely, $w_{1}, \delta$, and $w_{c \text {-Si }}$. The probability set $w_{1}=$ $0.01, \delta=10$, and $w_{c-\mathrm{Si}}=10$ was used throughout this work, as was used in our previous work on borosilicate glasses [12, 31, 32] and in the work of Devreux and co-workers [11, 26]. This probability set leads to good agreement with the silicon saturation concentration obtained in corrosion experiments of borosilicate glasses with varying boron content in alkaline conditions and at $90^{\circ} \mathrm{C}[26,35]$. 
In all the simulations presented in this work, the initial configuration of the model glass was that of a slab with a surface area of $64 \times 64$ sites and a thickness of 4096 or 8192 lattice sites. The slab thickness was selected to encompass the altered layer, with a thicker slab required for the glasses with a high B content as they develop thicker altered layers. The solution volume was set to be equal to $1.36453 \times 10^{10}$ aqueous sites, which translates to a surface-area-to-volume ratio of $1000 \mathrm{~m}^{-1}$, considering a lattice side length, $a$, of $0.3 \mathrm{~nm}$ for an individual lattice site [24].

In addition to dissolution (hydrolysis) and condensation reactions, an explicit representation of the diffusion of dissolved glass components in the altered glass layer is introduced in the Monte Carlo model. Because B and Na do not condense back on the glass surface in the MC model, only the diffusion of dissolved $\mathrm{Si}$ is considered in the simulations presented in this work. For the purposes of this work, the interface between the pristine glass and the altered layer is defined as the topmost full glass layer. Similarly, the interface between the altered layer and the bulk aqueous solution is defined as the topmost occupied glass layer. Three diffusion models have been implemented and are evaluated:

M1. Instantaneous homogenization of the aqueous solution after each dissolution event. This is the model that has been used in previous publications [12, 30-32]. It represents the limiting case where the diffusion coefficient of $\mathrm{Si}$ is very large compared to the rates of hydrolysis and condensation reactions, which results in a homogeneous distribution of dissolved Si species in the aqueous solution at each computer steps.

M2. The model proposed by Ledieu et al. [27], in which a linear Si concentration gradient is imposed across the altered layer and the Si concentration at depth $z$ and time $t, C_{\mathrm{Si}}(z, t)$ is defined as: 


$$
C_{\mathrm{Si}}(z, t)=C_{\mathrm{Si}}(0, t) \max \left(1+\frac{z-z_{0}(t)}{l_{d}} ; 1\right)
$$

where $C_{\mathrm{Si}}(0, t)$ is the concentration in the bulk aqueous solution, $z_{0}(t)$ is the depth of the topmost occupied glass layer, and $l_{d}$ is the diffusion length, which determines the slope of the concentration gradient.

M3. A model in which the hydrolysis and condensation reactions are coupled to a onedimensional (1D) diffusion model, as described in detail below.

Model M3 relies on a discrete version of Fick's $2^{\text {nd }}$ law

$$
\frac{\partial \phi}{\partial t}=D \frac{\partial^{2} \phi}{\partial z^{2}}
$$

solved on a 1D grid using the Crank-Nicolson method, a finite difference method that uses second-order central difference in space and trapezoidal rule in time. In Equation 2, $t$ is time, $z$ is the depth, $D$ is the silicon diffusion coefficient, and $\phi$ is $C_{\mathrm{Si}}(z, t)$. Each $x y$ layer of the 3D lattice represents one grid point on the $1 \mathrm{D}$ diffusion grid. The grid point spacing is set to be the lattice site length $a(\Delta z=a=0.3 \mathrm{~nm})$. The diffusion grid covers the entire altered layer, i.e. it is bounded by the topmost full glass layer and the topmost occupied glass layer. Instantaneous homogenization is assumed beyond the topmost occupied glass layer (in the bulk aqueous solution). Neumann boundary conditions are applied at both boundaries. To avoid spurious oscillations with the Crank-Nicolson method, the ratio $\Delta t D /(\Delta z)^{2}$, where $\Delta t$ is the integration time step, should not be greater than 0.5 . As a result, $\Delta t$ should be varied when changing $D$, since $\Delta z$ is fixed. Therefore, to allow for a consistent comparison between simulations with different values of $D$, the number of integration steps, $N$, performed at each MC step is set so that the integration time, $t_{\mathrm{int}}$, remains constant and equals the MC time step, $t_{\mathrm{MC}}\left(t_{\mathrm{int}}=t_{\mathrm{MC}}=N \Delta t\right)$. Based on a comparison between experimental and calculated corrosion kinetics of borosilicate glasses 
at $90^{\circ} \mathrm{C}$, Devreux et al. [26] determined a value of $2.5 \mathrm{~s}$ per computer step for the probability set used in this work, and therefore, $t_{\mathrm{MC}}$ was set to $2.5 \mathrm{~s}$ in this work. In diffusion model M3, the silicon diffusion coefficient is kept constant throughout the simulation.

The overall algorithm of the MC program is broken down as follows:

1. Dissolution evaluation and execution step: Each surface site is evaluated for dissolution using the dissolution probabilities given above.

2. Glass connectivity evaluation step: Clusters of lattice sites that are not connected to the main glass matrix are identified and dissolved.

3. Diffusion step: Concentration of dissolved glass components in the altered layer is updated based on the diffusion model described above.

4. Condensation step: The condensation of dissolved glass components at surface sites is evaluated using the condensation probabilities given above.

5. Liquid connectivity evaluation step: the connectivity of the water sites are evaluated in an analogous way to step 2 to identify those water sites that are connected to the bulk aqueous solution and those that belong to closed pores (as dissolution and condensation reactions are not considered in closed pores).

6. Coordination evaluation step: the coordination of each site is re-calculated for the new configuration of the glass slab.

\section{Results}

This section is divided into two subsections. Results obtained with the linear concentration gradient model M2 and the 1D finite difference model M3 are compared with those obtained 
with the reference instantaneous homogenization model M1 in the first and second subsections, respectively.

\subsection{Comparison of instantaneous homogenization (M1) and linear concentration gradient (M2)} models

MC simulations were carried out for three values of $l_{d}, 50,250$, and $500 \mathrm{~nm}$, and five values of $x$ resulting in five different $\mathrm{B}_{2} \mathrm{O}_{3}$ mol\% of $12.5,15,17.5,20$, and 22.5 to capture a broad range of possible conditions. Five simulations were performed for each $l_{d^{-}} x$ combination. The simulations were run for 1000000 (28.9 d), 600000 (17.4 d), and 300000 (8.7 d) computer steps (simulated time) for $l_{d}=50,250$, and $500 \mathrm{~nm}$, respectively. Different numbers of computer steps were used because smaller values of $l_{d}$ translate to longer times for reaching the Si saturation concentration in the bulk aqueous solution, as presented later in this section. Five simulations were also carried out for each value of $x$ and for 300000 computer steps (8.7 d) with model M1. Figure 1 (left panel) compares the changes in Si and B dissolved thicknesses obtained at the end of the simulations as a function of B content for models M1 and M2. The dissolved thickness of glass component $i, D T_{i}$, is calculated according to

$$
D T_{i}=\frac{a D_{i}}{N_{L} f_{i}}
$$

where $D_{i}$ is the final number of glass components $i$ dissolved in the aqueous solution, $f_{i}$ is the fraction of glass component $i$ in the pristine glass lattice, $N_{L}$ is the number of lattice sites in one pristine glass layer and $a$ is the lattice site length $(a=0.3 \mathrm{~nm})$.

The B dissolved thickness increases with increasing B content while the Si dissolved thickness is not as significantly influenced by the B content. As explained in previous publications [18, 24-26] for similar corrosion MC models, the kinetics of Si hydrolysis and 
condensation reactions lead to an increase in Si density and extent of polymerization in the altered layer over time. This phenomenon, which is more advanced in the outer region of the altered layer as this region has been in contact with the aqueous solution for a greater amount of time, results in the formation of a blocking layer that water cannot penetrate through. Eventually, the aqueous solution is in contact with a silicon layer of similar density for all compositions, which explains the lack of a strong dependence of the Si dissolved thickness on the B content. However, the forward dissolution rate increases significantly with increasing B content so that glasses with a high B content undergo a greater extent of corrosion before formation of the blocking layer, which translates to a strong dependence of the B dissolved thickness on the B content of the pristine glass.
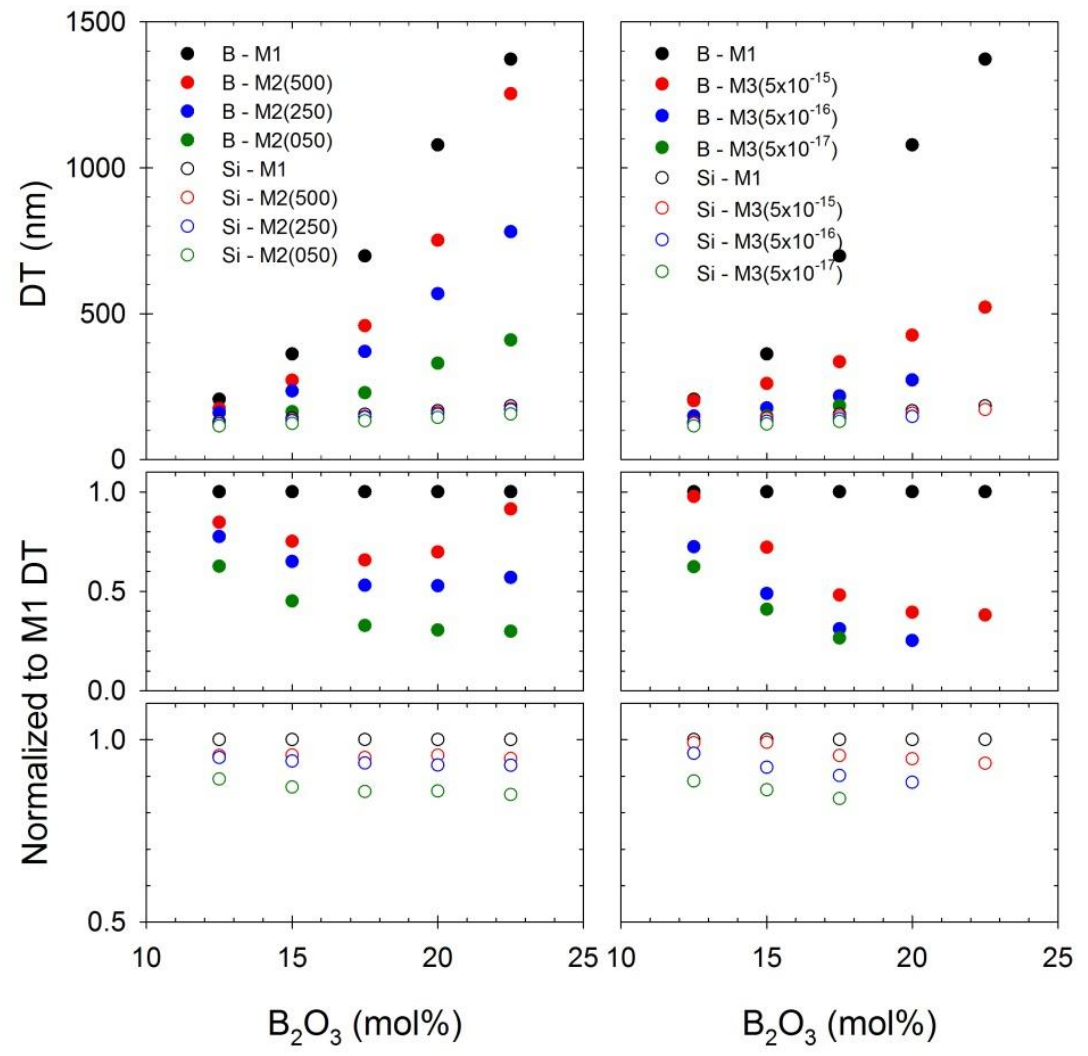

Figure 1. (top) Si (open circles) and B (closed circles) dissolved thicknesses obtained with 
models M2 (left) and M3 (right) as a function of B content and for three values of $l_{d}$ and $D$, as shown in brackets. Results obtained with M1 are shown for comparison. (middle) B dissolved thickness normalized to that obtained with M1 for the same B content. (bottom) Si dissolved thickness normalized to that obtained with M1 for the same B content. Error bars are smaller or commensurate with data points.

The greater the value of $l_{d}$, the lower the concentration gradient and thus the closer the Si concentration is to the homogenous concentration case (see Equation 1). Conversely, decreasing values of $l_{d}$ increase the concentration gradient and thus increasingly favor condensation at the interface between the altered layer and the pristine glass. This process accelerates $\mathrm{Si}$ polymerization, with the largest effect near the interface between the altered layer and the pristine glass. As a result, the formation of a blocking layer occurs earlier, leading to thinner altered layers and thus smaller B dissolved thicknesses, as shown in Figure 1 (left panel). These results are in agreement with those obtained by Ledieu et al. [27] with the same model for a glass with 20 mol\% $\mathrm{B}_{2} \mathrm{O}_{3}$. Normalization to the dissolved thicknesses obtained with model M1 shows that the Si dissolved thickness is less influenced by the value of $l_{d}$ than the B dissolved thickness and that it shows little to no dependence on the B content, for reasons explained above. However, the normalized B dissolved thickness initially decreases with increasing B content and subsequently plateaus or even increases slightly at high B content. The increase in dissolved Si at depth $z$ in Equation 1 is dependent on the altered layer thickness so that, as the B content increases, the altered layer becomes thicker and the concentration gradient is also increased.

In model M1, as noted above, the outer region of the glass has been in contact with the bulk solution for the greatest amount of time; therefore, the extent of $\mathrm{Si}$ polymerization is more advanced in this region. This phenomenon leads to a Si occupancy profile shown in Figure 2 (top 
panel), for a glass with $17.5 \mathrm{~mol} \% \mathrm{~B}_{2} \mathrm{O}_{3}$, whereby the $\mathrm{Si}$ density is lower than that of the pristine glass near the interface with the pristine glass and higher than the pristine glass near the interface with the aqueous solution. In contrast, condensation near the interface with the pristine glass is favored in model M2, which leads to a shrinking low density region as the value of $l_{d}$ decreases. As a result, the porosity of the altered layer is lower and more homogeneous in the presence of a concentration gradient. Again, these results are in good agreement with those reported by Ledieu et al. [27]. In model M1, variation of the B content leads to increasing differences between the maximum and minimum of the Si occupancy in the altered layer (relative to that in the pristine glass) with increasing B content (Figure 3). In model M2, the low-density region is greatly suppressed at all B contents, whereas the maximum in Si occupancy is little affected.
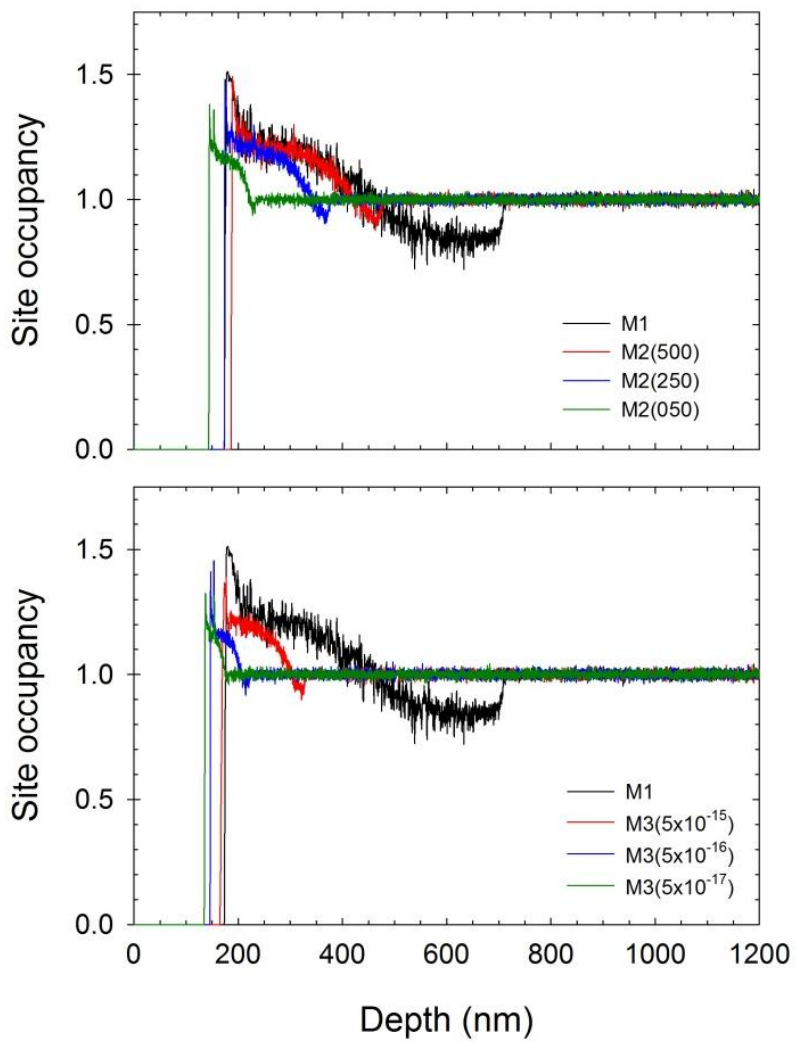

Figure 2. Site occupancy by Si sites as a function of depth at the end of the MC simulations for a glass of composition $65 \mathrm{~mol} \% \mathrm{SiO}_{2} 17.5 \mathrm{~mol} \% \mathrm{~B}_{2} \mathrm{O}_{3} 17.5 \mathrm{~mol} \% \mathrm{Na}_{2} \mathrm{O}$. The profile obtained 
with model M1 is compared to those obtained with models M2 (top), and M3 (bottom) with values of $l_{d}$ and $D$ shown in brackets. The initial position of the interface between the glass surface and the aqueous solution is used as the reference depth. The site occupancy is the number of Si sites per unit area in each $x y$ layer normalized to that in the pristine glass.
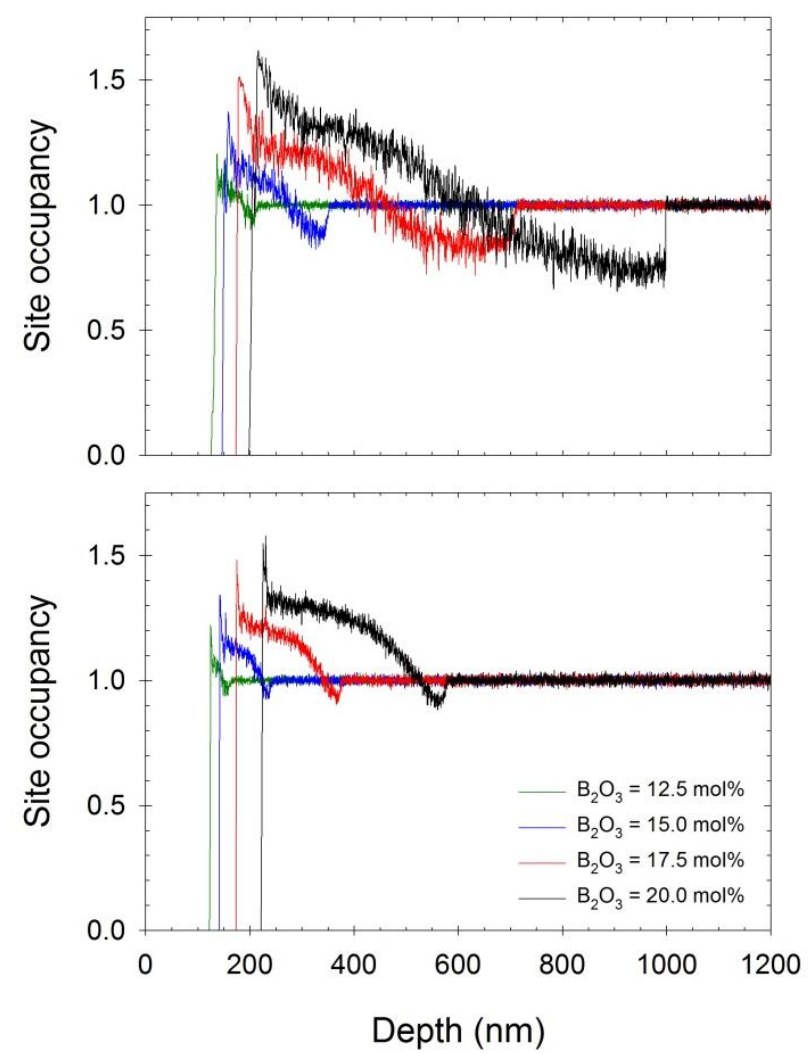

Figure 3. Site occupancy by Si sites as a function of depth at the end of the MC simulations for glasses with $\mathrm{B}_{2} \mathrm{O}_{3}$ contents of $12.5,15.0,17.5$, and 20.0 mol\% as obtained with model M1 (top) and M2 $\left(l_{d}=250 \mathrm{~nm}\right)$ (bottom). The initial position of the interface between the glass surface and the aqueous solution is used as the reference depth. The site occupancy is the number of $\mathrm{Si}$ sites per unit area in each $x y$ layer normalized to that in the pristine glass.

3.2. $1 D$ finite difference (M3) model and comparison with models M1 and M2 
MC simulations were carried out for three values of $D$ in Equation 2, $5 \times 10^{-15}, 5 \times 10^{-16}$, and $5 \times 10^{-17} \mathrm{~m}^{2} \mathrm{~s}^{-1}$, and the same five values of $x$. As explained in the Computational Methods section, high values of $D$ require large numbers of integration steps (i.e. the number of times the 1D diffusion equation is solved per computer step) because of the need to keep the integration time step small enough to avoid spurious oscillations in the finite difference method. As a result, the upper value of $D$ was limited by computational expense. The lower value of $D$ was limited by the point where the B dissolved thickness falls to that of $\mathrm{Si}$. For the simulations where $D=5 \times 10^{-15}$ $\mathrm{m}^{2} \mathrm{~s}^{-1}, 2$ simulations were performed for 300000 (8.7 d) computer steps (simulated time), whereas, for the other two values of $D, 3$ simulations were performed for 1000000 (28.9 d) computer steps (simulated time). As will be explained later in this section, lower values of $D$ translate to longer times for the bulk aqueous solution to reach the Si saturation concentration, which is why a higher number of computer steps was used for the lower values of $D$.

Figure 1 (right panel) compares the changes in $\mathrm{Si}$ and $\mathrm{B}$ dissolved thicknesses obtained at the end of the simulations as a function of B content for models M1 and M3. Overall, the behavior of model M3 is similar to that of M2 whereby a decrease in the Si diffusion coefficient translates to a thinner B dissolved thickness but has little effect on the Si dissolved thickness. Similarly, the B dissolved thickness relative to that obtained with M1 for the same B content shows the same trend as observed for model M2. Finally, there is also good agreement between models M2 and M3 in terms of the Si site occupancy profiles (Figure 2) whereby the reduced diffusion in model M3 leads to a suppression of the low-density region in the altered layer near the interface with the pristine glass.

Because model M2 is more rapid to execute than model M3 and is less computationally expensive, it is encouraging to observe that the simple diffusion model described by Equation 1 
can reproduce important features of the more complex diffusion model that requires the explicit solution of diffusion equations. However, there are critical differences between the three models that are not evident in the values of the dissolved thicknesses. Indeed, the time evolution of the altered layer is significantly different for the three models, as illustrated in Figure 4 for a glass of composition $65 \mathrm{~mol} \% \mathrm{SiO}_{2} 17.5 \mathrm{~mol} \% \mathrm{~B}_{2} \mathrm{O}_{3} 17.5 \mathrm{~mol} \% \mathrm{Na}_{2} \mathrm{O}$.
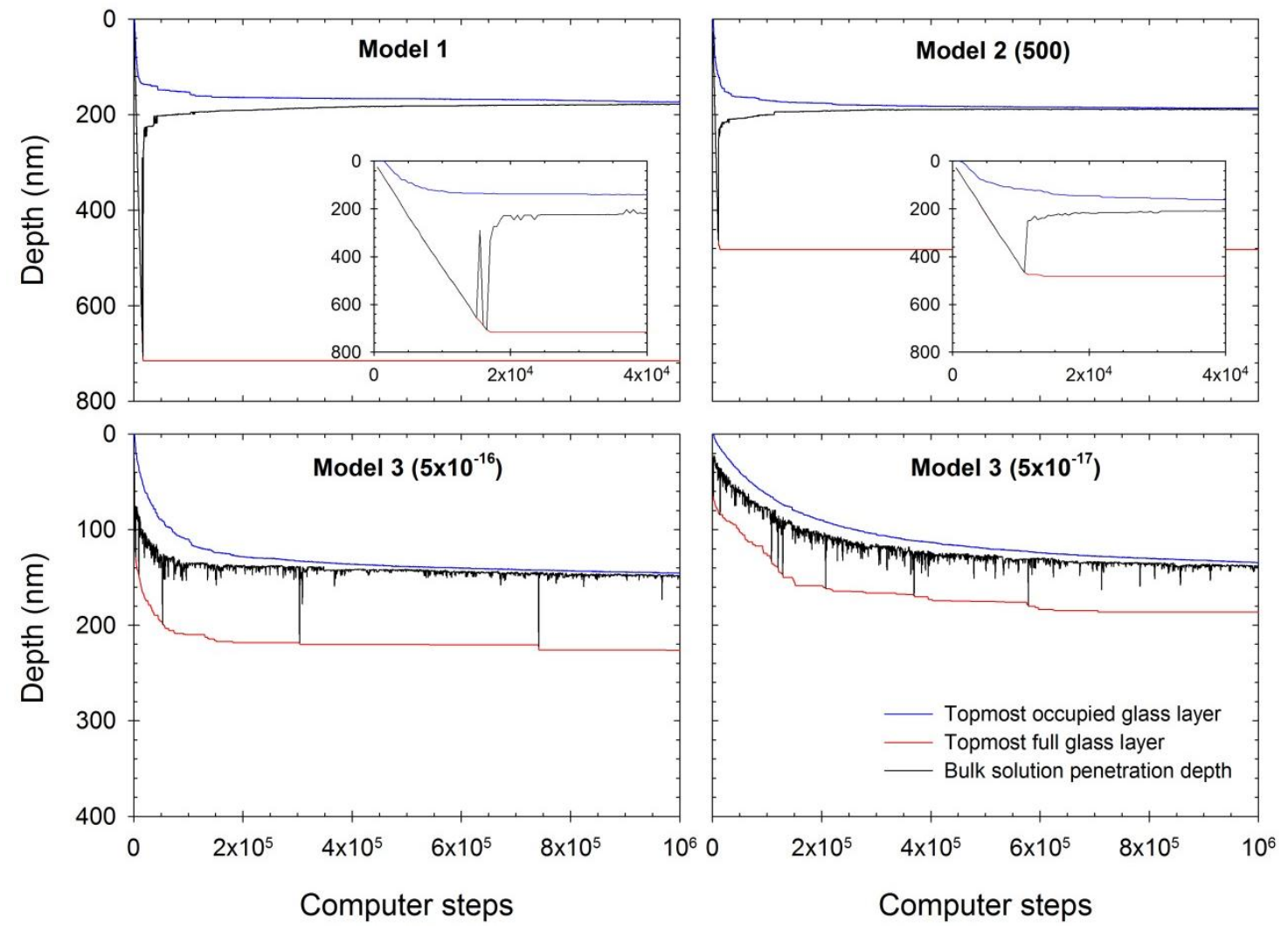

Figure 4. Depth profiles showing the positions of the topmost occupied and topmost full glass layers as a function of computer steps for MC simulations performed with models M1, M2, and M3 (values of $l_{d}$ and $D$ are shown in brackets) for a glass of composition $65 \mathrm{~mol} \% \mathrm{SiO}_{2} 17.5$ mol\% $\mathrm{B}_{2} \mathrm{O}_{3} 17.5 \mathrm{~mol} \% \mathrm{Na}_{2} \mathrm{O}$. Also shown are the depths to which the bulk aqueous solution can penetrate. Insets in the top panels show a close-up of the first 40000 computer steps.

Figure 4 shows that, for model M1, there is an initial period during which the altered layer thickness increases and the bulk solution can penetrate through to the interface with the pristine 
glass. Then, as the outer region of the altered layer polymerizes and becomes denser, a layer forms that blocks water from penetrating through to the interface with the pristine glass. For model M2, the behavior of the altered layer is similar except that the onset of the formation of the blocking layer occurs earlier, which results in a thinner altered layer. For model M3 however, a very different picture emerges. Clogging of the pores in the altered layer occurs very early on, but water occasionally breaks through and penetrates through to the interface with the pristine glass again. As a result, the time needed to form a stable blocking layer with model M3 is greatly delayed. This effect is accentuated by lower values of the diffusion coefficient (Figure 4).
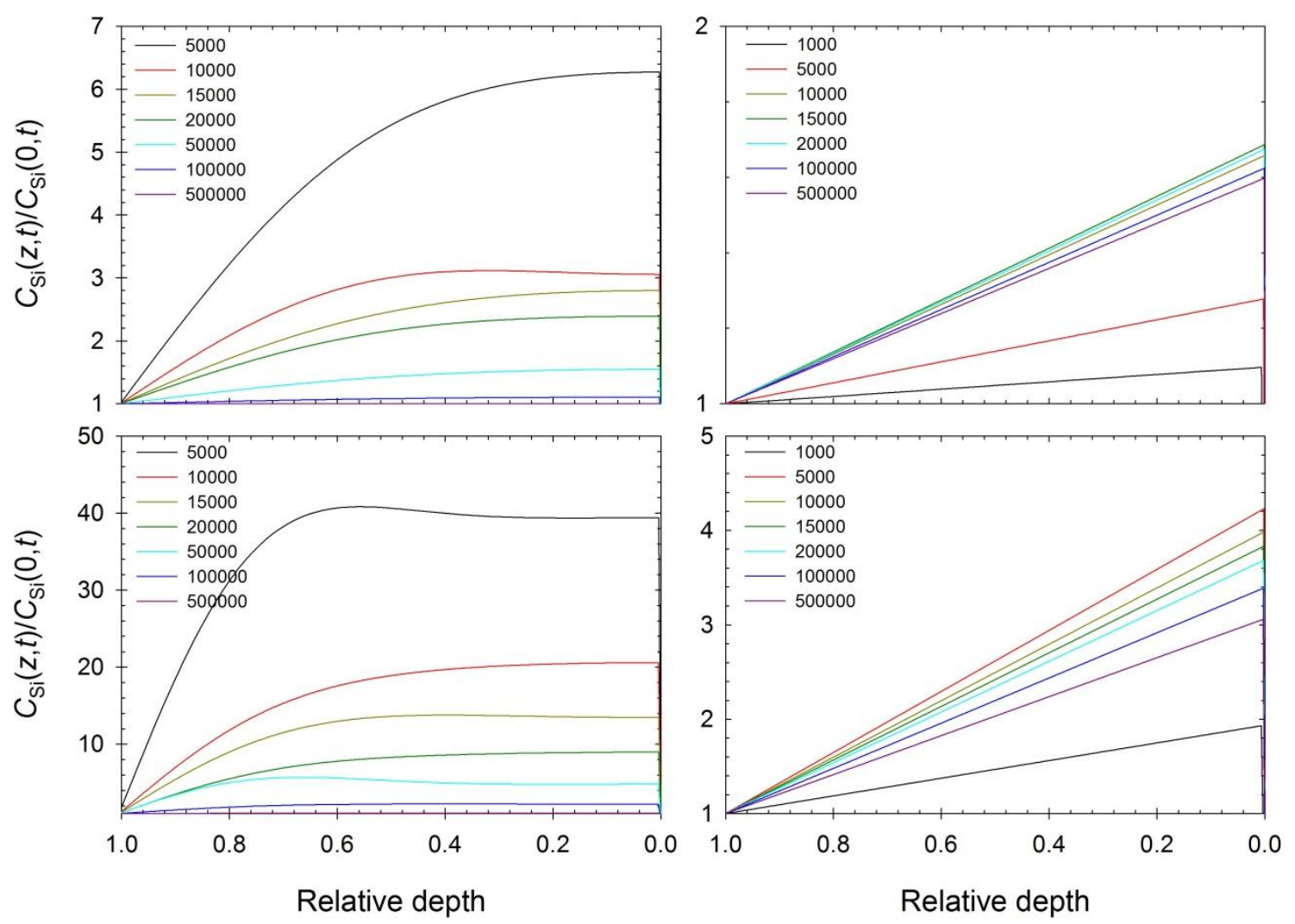

Figure 5. Dissolved Si concentration in the altered layer relative to that in the bulk aqueous solution as a function of relative depth, where 1.0 is the position of the topmost occupied layer and 0.0 is the position of the topmost full glass layer, as obtained with model M3 with $D=5 \times 10^{-}$ 
${ }^{16} \mathrm{~m}^{2} \mathrm{~s}^{-1}$ (top left) and $D=5 \times 10^{-17} \mathrm{~m}^{2} \mathrm{~s}^{-1}$ (bottom left) and model M2 with $l_{d}=500 \mathrm{~nm}$ (top right) and $l_{d}=50 \mathrm{~nm}$ (bottom right) at different points along the course of the simulations (in computer steps) for a glass of composition $65 \mathrm{~mol} \% \mathrm{SiO}_{2} 17.5 \mathrm{~mol} \% \mathrm{~B}_{2} \mathrm{O}_{3} 17.5 \mathrm{~mol} \% \mathrm{Na}_{2} \mathrm{O}$.

This significant difference between models M2 and M3 stems from the difference in the time evolution of the dissolved Si concentration profile in the altered layer predicted by the two models. According to Equation 1, as the glass begins to dissolve and the altered layer increases in thickness (Figure 4), the linear concentration gradient initially becomes steeper with time with model M2 (Figure 5). Once the blocking layer has formed and the depth of the topmost occupied layer decreases towards a plateau value (Figure 4), the concentration gradient decreases slightly (Figure 5). With model M3, a different phenomenon is observed: the initial high flux of dissolving Si cannot diffuse rapidly through the altered layer leading to a significant extent of Si accumulation, whereas, as the net rate of the forward reaction slows down with time, the gradient of concentration between the deepest region of the altered layer and the bulk aqueous solution diminishes (Figure 5). Consequently, the clogging of pores occurs early on with model M3; but by extensive condensation of poorly polymerized Si sites rather than by the gradual formation of a highly polymerized layer as is the case with models M1 and M2. Consequently, hydrolysis of the pore walls is more facile and the porous structure of the altered layer is much more dynamic with model M3.

This difference in pore structure dynamics leads to different dissolution kinetics. Figure 6 shows the time evolution of the dissolved $\mathrm{Si}$ and B concentrations in the bulk aqueous solution. For model M1 and for high values of $l_{d}$ with model M2, the dissolved Si concentration rapidly reaches equilibrium, whereas equilibrium is significantly postponed for low values of $l_{d}$ and for model M3. More strikingly, the evolution of the dissolved B concentration calculated with model 
M3 differs significantly from that obtained with models M1 and M2. For example, even though a value of $l_{d}$ of $50 \mathrm{~nm}$ leads to a dissolved B concentration commensurate with that obtained for a value of $D$ of $5 \times 10^{-16} \mathrm{~m}^{2} \mathrm{~s}^{-1}$, the kinetics of dissolution are slower for model M3. In addition, after a short period of rapid $\mathrm{B}$ release, model $\mathrm{M} 3$ predicts the release of $\mathrm{B}$ at a greatly diminished rate, unlike what is obtained in models M1 and M2.

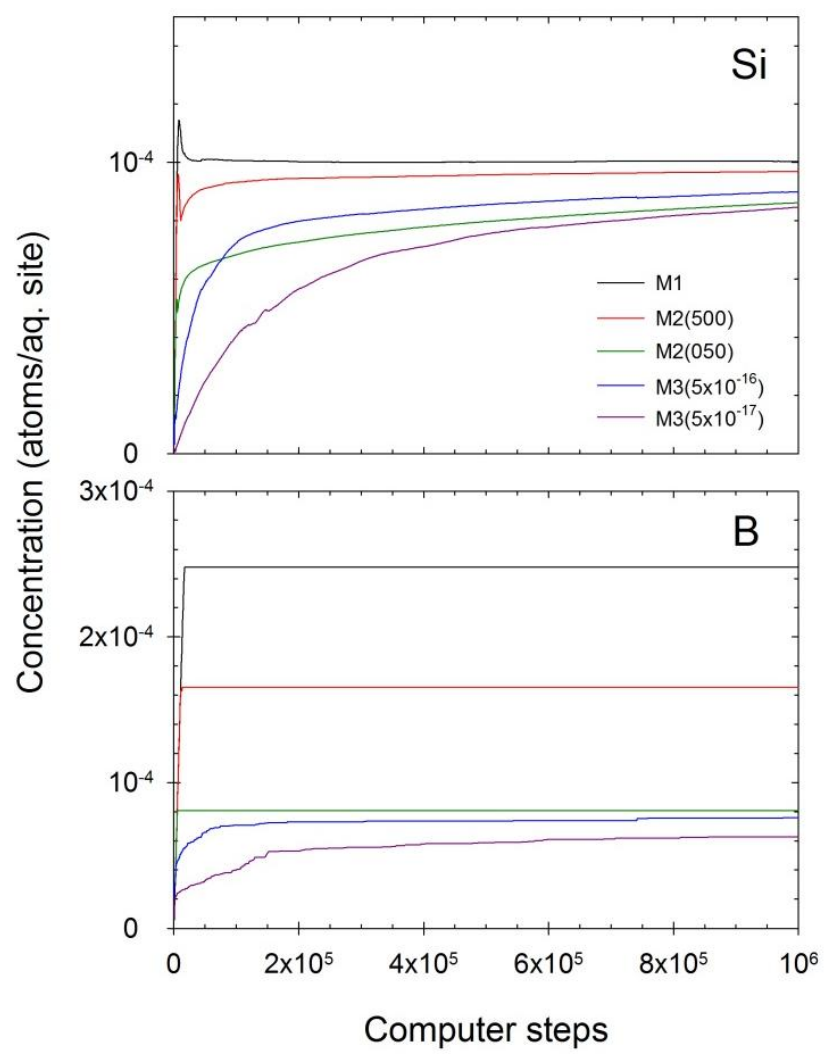

Figure 6. (top) Silicon and (bottom) B concentrations in the bulk aqueous solution as a function of computer steps for MC simulations performed with models M1, M2, and M3 (values of $l_{d}$ and $D$ are shown brackets) for a glass of composition $65 \mathrm{~mol} \% \mathrm{SiO}_{2} 17.5 \mathrm{~mol} \% \mathrm{~B}_{2} \mathrm{O}_{3} 17.5 \mathrm{~mol} \%$ $\mathrm{Na}_{2} \mathrm{O}$.

\section{Discussion}


The diffusion models evaluated in this work all show a decreasing B dissolved thickness as the rate of diffusion (or diffusion length for M2) is reduced, with the effects of diffusion being more significant for glasses that develop a thick altered layer. However, the different diffusion models also show marked differences in terms of the predicted alteration layer thicknesses and morphologies and, importantly, in terms of the kinetics of corrosion and time evolution of the dissolved Si concentration in the altered layer. In this section, these findings are compared and contrasted with experimental observations and other models of borosilicate glass corrosion. First, a quantitative comparison is presented with experimental dissolution rates obtained by Jégou et al. [36] and Gin et al. [37] for a glass with a composition within the range of simulated compositions. Second, a qualitative comparison is provided with the kinetics of dissolution obtained by Rebiscoul et al. [8] for three glasses. The study of Rebiscoul et al. [8] offers a suitable set of data for comparison with the MC simulations, as it provides B release kinetics as well as altered layer thicknesses and morphologies for three glasses with notably different behaviors.

Jégou et al. [36] and Gin et al. [37] measured the rate of dissolution of CJ1 (67.7 mol\% $\mathrm{SiO}_{2}$ $18.0 \mathrm{~mol} \% \mathrm{~B}_{2} \mathrm{O}_{3} 14.2 \mathrm{~mol} \% \mathrm{Na}_{2} \mathrm{O}$ ) at $90{ }^{\circ} \mathrm{C}$ and initial $\mathrm{pH}=9$ and at surface-area-to-volume ratios of 10,400 , and $8000 \mathrm{~m}^{-1}$. Dissolution at $10 \mathrm{~m}^{-1}$ was congruent over the first 48 hours and the initial dissolution rate, $r_{0}$, obtained from this period was $4.5 \pm 1 \mathrm{~g} \mathrm{~m}^{-2} \mathrm{~d}^{-1}$. Initial dissolution rates obtained for the two glasses with closest composition, i.e. $x=5$ (70 $\mathrm{mol}^{2} \mathrm{SiO}_{2} 15 \mathrm{~mol} \%$ $\left.\mathrm{B}_{2} \mathrm{O}_{3} 15 \mathrm{~mol} \% \mathrm{Na}_{2} \mathrm{O}\right)$ and $x=10\left(65 \mathrm{~mol} \% \mathrm{SiO}_{2} 17.5 \mathrm{~mol} \% \mathrm{~B}_{2} \mathrm{O}_{3} 17.5 \mathrm{~mol} \% \mathrm{Na}_{2} \mathrm{O}\right)$, were 1.7 and $5.9 \mathrm{~g} \mathrm{~m}^{-2} \mathrm{~d}^{-1}$, respectively. Therefore, the calculated dissolution rates are consistent with and bracket the experimental dissolution rate. The two reported initial dissolution rates were calculated with model M1 for the initial regime of B linear release (see Figure 6 for example). 
Model M1 had the longest period of linear release, which facilitated the determination of the initial dissolution rate, but the other models showed similar initial rates, albeit over shorter periods. Jégou et al. [36] reported a decrease in the dissolution rate of about one order of magnitude $\left(0.35 \mathrm{~g} \mathrm{~m}^{-2} \mathrm{~d}^{-1}\right)$ over the period between 7 and 14 days at $400 \mathrm{~m}^{-1}$. As presented in the Results section and illustrated in Figures 4 and 6, B release is stopped with models M1 and M2 once the blocking layer is formed; however, the altered layer is more dynamic with model M3, which leads to intermittent release of B. Therefore, a finite dissolution rate based on B release over the period between 7 and 14 days was only calculated for model M3. The values thus obtained were $1.1 \times 10^{-3}$ and $9.0 \times 10^{-3} \mathrm{~g} \mathrm{~m}^{-2} \mathrm{~d}^{-1}$ for $x=5$ and $D=5 \times 10^{-16}$ and $5 \times 10^{-17} \mathrm{~m}^{2} \mathrm{~s}^{-1}$, respectively, and $8.2 \times 10^{-4}$ and $5.9 \times 10^{-3} \mathrm{~g} \mathrm{~m}^{-2} \mathrm{~d}^{-1}$ for $x=10$ and $D=5 \times 10^{-16}$ and $5 \times 10^{-17} \mathrm{~m}^{2} \mathrm{~s}^{-1}$, respectively. In other words, the MC simulations suggest a decrease in the dissolution rate of approximately 2 to 3 orders of magnitude relative to the initial rate. The difference between the calculated and experimental dissolution rates could be due to the higher surface-area-to-volume ratio used in the simulations. However, the calculated dissolution rates are also slightly lower than that reported by Gin et al. [37] $\left(1.6 \times 10^{-2} \mathrm{~g} \mathrm{~m}^{-2} \mathrm{~d}^{-1}\right)$ for CJ1 after one year of corrosion at $8000 \mathrm{~m}^{-1}$. In the MC model, B is only released from the glass when water is able to break through the blocking layer. If $\mathrm{B}$ was allowed to diffuse through the dense region of the altered layer, this could lead to higher release rates that would be in better agreement with those derived experimentally. Future MC models will investigate this potential mechanism.

Rebiscoul et al. [8] studied the changes in altered layer morphology of three glasses corroded in static, aqueous conditions using X-ray reflectometry (XRR). The three glasses were SON68 and two simplified 5-oxide glasses with the same molar ratios as SON68 (Zirconiumfree Simplified Glass 1 (mol\%): $61.2 \% \mathrm{SiO}_{2} 3.9 \% \mathrm{Al}_{2} \mathrm{O}_{3} \quad 16.3 \% \mathrm{~B}_{2} \mathrm{O}_{3} 12.8 \% \mathrm{Na}_{2} \mathrm{O} 5.8 \% \mathrm{CaO}$; 
Aluminum-free Simplified Glass 2 (mol\%): $62.5 \% \mathrm{SiO}_{2} \quad 16.6 \% \mathrm{~B}_{2} \mathrm{O}_{3} \quad 13.1 \% \mathrm{Na}_{2} \mathrm{O} \quad 6.0 \% \mathrm{CaO}$ $\left.1.8 \% \mathrm{ZrO}_{2}\right)$. The total mole percentage of insoluble or sparingly soluble oxides $\left(\mathrm{SiO}_{2}, \mathrm{Al}_{2} \mathrm{O}_{3}\right.$, and $\mathrm{ZrO}_{2}$ ) in Simplified Glass 1 and 2 are $65.1 \%$ and $64.3 \%$, respectively; therefore, the most appropriate composition for comparison is the simulated borosilicate glass with $65 \% \mathrm{SiO}_{2}$.

Rebiscoul et al. reported very different time evolution of the B dissolved thickness for these three glasses. Glasses 2 altered very rapidly but B release stopped abruptly after a short period of time. This phenomenon has been consistently observed for simple borosilicate glasses containing up to a few mole percent $\mathrm{ZrO}_{2}$ and corroded in neutral to alkaline conditions $[10,29]$ and has been reproduced by Monte Carlo simulations [10, 24, 29]. As explained in the Results section, the Monte Carlo simulations, including those performed in this work with models M1 and M2, demonstrate the origin of this phenomenon; specifically, the formation of a dense layer in the outer region of the altered layer that prevents water from penetrating through to the pristine glass. Transmission electron microscopy images, a combination of small-angle X-ray scattering and neutron scattering [10] as well as the XRR data of Rebiscoul et al. all provided supporting evidence for this interpretation. Glass 1 , which contains $\mathrm{Al}_{2} \mathrm{O}_{3}$ rather than $\mathrm{ZrO}_{2}$, shows a different behavior whereby the initial B release is slower and the change in B dissolved thickness exhibits a gradual decrease, although its B dissolved thickness eventually reaches that of Glass 2. This is similar to the differences observed between the B dissolved thicknesses calculated with models M2 $\left(l_{d}=50 \mathrm{~nm}\right)$ and M3 $\left(D=5 \times 10^{-16} \mathrm{~m}^{2} \mathrm{~s}^{-1}\right)$. The initial B release rate with model M2 $\left(l_{d}=50 \mathrm{~nm}\right)$ is fast and the same as that obtained with other values of $l_{d}$ as well as with model M1, with the differences in final $\mathrm{B}$ dissolved thicknesses due to the time required for forming the blocking layer. B release as calculated by model M3 $\left(D=5 \times 10^{-16} \mathrm{~m}^{2} \mathrm{~s}^{-1}\right)$ is initially slower and 
slows down further with time, although the dissolved B concentration eventually equals that calculated by model M2 $\left(l_{d}=50 \mathrm{~nm}\right)$, as shown in Figure 6.

In addition, the XRR data of Rebiscoul et al. indicate a different altered layer morphology for Glass 1 with the presence of a thin dense layer sandwiched between the pristine glass and a thick gel layer; although the density difference between the two layers is not as extensive as for Glass 2. The reduction and eventual disappearance of the low-density region of the altered layer in the MC simulations carried out with model M3 and model M2 with low values of $l_{d}$ are consistent with this experimental observation. However, the simulations do not predict the formation of a thick gel layer with reduced density atop the dense layer. This difference could be due in part to the fact that the analysis of the XRR data was done assuming two layers atop the substrate whereas the MC simulations are able to capture gradual changes with a $0.3 \mathrm{~nm}$ resolution. It should also be noted that the MC simulations consider a simplified glass structure mapped onto a perfect lattice that does not account for any extended structural and topographical heterogeneities of the glass. The discrepancy between experimental and calculated gel thicknesses could be due to a combination of both issues as well as to the fact that the glasses considered in the MC simulations did not contain aluminum.

The evolution of the B dissolved thickness obtained with model M3 $\left(D=5 \times 10^{-17} \mathrm{~m}^{2} \mathrm{~s}^{-1}\right)$ matches well that obtained for SON68 with a rapid rise followed by a more linear, slow increase. Additionally, the XRR data indicate a much thinner altered layer for SON68 compared to Simplified Glasses 1 and 2, which is consistent with the fact that the MC simulations show a thinner altered layer for the lower diffusion coefficients with model M3.

In summary, the MC simulations provide new insights into the likely processes that underlie the differences between the three glasses studied by Rebiscoul et al. [8]. Diffusion in the altered 
layer of the $\mathrm{ZrO}_{2}$-containing glass is facile, which allows the interstitial aqueous solution to homogenize rapidly. In turn, this allows for extensive polymerization of the altered layer and the eventual formation of a dense, blocking layer in its outer region. For the $\mathrm{Al}_{2} \mathrm{O}_{3}$-containing glass, diffusion through the pores is slower, leading to a build-up of dissolved Si in the pores and more extensive condensation in the altered layer in the early stages of corrosion. This effect interferes with the gradual strengthening of pore walls so that pores close and open frequently and thus B is released at a much lower rate but over a longer period of time. For SON68, diffusion is even slower, the altered layer is thin and dense, and the rate of B release diminishes greatly as a result.

Leaving aside the formation of secondary phases, which was not considered in this work, the behavior of model M3 has similarities with that of the passivating reactive interphase (PRI), which is at the heart of the GRAAL model $[38,39]$. This model assumes the formation of a layer at the interface between the pristine glass and the altered layer that strongly inhibits diffusion and thus controls corrosion of the pristine glass. The PRI is created at a rate proportional to the ratio of the diffusion coefficient of water through it and its thickness and dissolves following a firstorder affinity law. The GRAAL model presumes the presence of a gel depleted in Si and other elements atop the PRI but assumes that this gel does not affect the kinetics of corrosion. Model M3 is consistent with the PRI model, i.e. it shows that slow diffusion through even a thin alteration layer can significantly inhibit alteration kinetics and that the thickness of the PRI/diffusion layer diminishes for decreasing diffusion coefficients

Finally, model M2 assumes that diffusion through the altered layer results in a dissolved Si concentration profile linear with depth. This assumption is similar to that adopted by some models of nuclear waste glass dissolution such as the LIXIVER model [40]. However, model M3, in which the Si concentration profiles are obtained from the coupling of hydrolysis and 
condensation reactions with diffusion through the altered layer, clearly shows that the variations in concentration with depth are not only more complex than a linear behavior but are also likely to change with time.

\section{Conclusions}

Three different models for the diffusion of dissolved Si species in the altered layer of borosilicate glasses were evaluated systematically as a function of diffusion parameters (i.e. diffusion length, $l_{d}$, and diffusion coefficient, $D$ ) and glass composition. Model M1 assumed instantaneous homogenization of the dissolved species in the altered layer and bulk aqueous solution; model M2 made use of a linear concentration gradient to represent the distribution of dissolved Si species in the altered layer; and model M3 determined the Si concentration profile at each MC step by solving Fick's $2^{\text {nd }}$ law.

Comparison between the results obtained with the three models as well as with experimental data on the thickness and morphology of the altered layers of similar glasses revealed that the time evolution of the altered layer predicted by models M1 and M2 are appropriate for borosilicate glasses that are observed to dissolve rapidly such as sodium borosilicate glasses with low $\mathrm{ZrO}_{2}$ content but not for more complex and slow dissolving glasses such as SON68. Comparison between the Si concentration profiles in the altered layer obtained with models M2 and M3 highlighted issues with the assumption of a linear concentration profile of constant gradient. Specifically, explicitly solving Fick's $2^{\text {nd }}$ law yields concentration profiles in the altered layer that are not linear, change significantly with time, and decrease the magnitude of the concentration gradient with respect to the bulk aqueous solution as corrosion progresses. Therefore, explicit transport of dissolved Si in the altered layer, or a simplified model that can be 
more rapidly executed but yet reproduces the main features of the explicit model, should be used in simulations of more slowly dissolving nuclear waste glasses.

Future model development should consider the mechanisms that lead to the formation of a thick gel atop a dense layer rich in Si and insoluble oxides, as is observed for several nuclear waste glasses. The use of glass structures determined from molecular dynamics simulations [14, $32,41]$ should provide an improvement over the regular lattices used to date in MC models. The use of bond strengths determined from ab initio calculations [15] would also allow the extension of the number of oxide components considered without the need for parameterization from experimental data.

\section{Acknowledgements}

This work was funded by the Office of Nuclear Energy (Fuel Cycle Research and Development) and the Office of Environmental Management (Tank Waste Management, EM-21) of the U.S. Department of Energy (DOE). Some computer simulations were performed as part of a DOE Office of Science-supported Science Theme User Proposal at the William R. Wiley Environmental Molecular Sciences Laboratory (EMSL) Molecular Science Computing (MSC) facilities. The EMSL is a national scientific user facility sponsored by the Office of Science's Office of Biological and Environmental Research (OBER) and located at Pacific Northwest National Laboratory (PNNL). PNNL is operated for the DOE by Battelle Memorial Institute under Contract DE-AC05-76RL01830. Oak Ridge National Laboratory (ORNL) is managed by UT-Battelle LLC for the DOE under contract DE-AC05-00OR22725. 


\section{References}

[1] J.F. Ahearne, Phys. Today, 50 (1997) 24-29.

[2] R.C. Ewing, Elements, 2 (2006) 331-334.

[3] S. Gin, A. Abdelouas, L.J. Criscenti, W.L. Ebert, K. Ferrand, T. Geisler, M.T. Harrison, Y. Inagaki, S. Mitsui, K.T. Mueller, J.C. Marra, C.G. Pantano, E.M. Pierce, J.V. Ryan, J.M.

Schofield, C.I. Steefel, J.D. Vienna, Mater. Today, 16 (2013) 243-248.

[4] B. Grambow, Elements, 2 (2006) 357-364.

[5] J.D. Vienna, J.V. Ryan, S. Gin, Y. Inagaki, Int. J. Appl. Glass Sci., 4 (2013) 283-294.

[6] S. Gin, J.V. Ryan, D.K. Schreiber, J. Neeway, M. Cabié, Chem. Geol., 349-350 (2013) 99109.

[7] S. Gin, C. Guittonneau, N. Godon, D. Neff, D. Rebiscoul, M. Cabié, S. Mostefaoui, J. Phys. Chem. C, 115 (2011) 18696-18706.

[8] D. Rebiscoul, A. van der Lee, F. Rieutord, F. Né, O. Spalla, A. El-Mansouri, P. Frugier, A. Ayral, S. Gin, J. Nucl. Mater., 326 (2004) 9-18.

[9] K.A. Murphy, N.M. Washton, J.V. Ryan, C.G. Pantano, K.T. Mueller, J. Non-Cryst. Solids, 369 (2013) 44-54.

[10] C. Cailleteau, F. Angeli, F. Devreux, S. Gin, J. Jestin, P. Jollivet, O. Spalla, Nature Mater., 7 (2008) 978-983.

[11] A. Ledieu, F. Devreux, P. Barboux, Y. Minet, Nucl. Sci. Eng., 153 (2006) 285-300.

[12] S. Kerisit, E.M. Pierce, J. Non-Cryst. Solids, 358 (2012) 1324-1332.

[13] M. Aertsens, D. Ghaleb, J. Nucl. Mater., 2001 (2001) 37-46.

[14] J.M. Delaye, A. Kerrache, S. Gin, Chem. Phys. Lett., 588 (2013) 180-183.

[15] P. Zapol, H. He, K.D. Kwon, L.J. Criscenti, Int. J. Appl. Glass Sci., 4 (2013) 395-407. 
[16] M. Aertsens, P. Van Iseghem, Mater. Res. Soc. Symp. Proc., 412 (1996) 271-278.

[17] M. Aertsens, Modelling of glass dissolution with a Monte Carlo method. , in: Proc. 1998 CEA Valrho Summer Workshop on Glass: Scientific Research for High Performance Containment, 1998, pp. 343-354.

[18] M. Aertsens, Mater. Res. Soc. Symp. Proc., 556 (1999) 409-419.

[19] M. Aertsens, Ceramics-Silikaty, 43 (1999) 175-180.

[20] B. Grambow, Mat. Res. Soc. Symp. Proc., 44 (1985) 15-27.

[21] B. Grambow, R. Müller, J. Nucl. Mater., 298 (2001) 112-124.

[22] S.B. Santra, B. Sapoval, P. Barboux, F. Devreux, C. R. Acad. Sci. Paris, 215 (1998) 129134.

[23] F. Devreux, P. Barboux, J. Nucl. Mater., 298 (2001) 145-149.

[24] F. Devreux, P. Barboux, M. Filoche, B. Sapoval, J. Mater. Sci., 36 (2001) 1331-1341.

[25] M. Lobanova, L. Maurer, P. Barboux, F. Devreux, Y. Minet, Mater. Res. Soc. Symp. Proc., 663 (2001) 237-245.

[26] F. Devreux, A. Ledieu, P. Barboux, Y. Minet, J. Non-Cryst. Solids, 343 (2004) 13-25.

[27] A. Ledieu, F. Devreux, P. Barboux, J. Non-Cryst. Solids, 345\&346 (2004) 715-719.

[28] M. Arab, C. Cailleteau, F. Angeli, F. Devreux, L. Girard, O. Spalla, J. Non-Cryst. Solids, 354 (2008) 155-161.

[29] C. Cailleteau, F. Devreux, O. Spalla, F. Angeli, S. Gin, J. Phys. Chem. C, 115 (2011) 58465855.

[30] S. Kerisit, E.M. Pierce, Geochim. Cosmochim. Acta, 75 (2011) 5296-5309.

[31] S. Kerisit, J.V. Ryan, E.M. Pierce, J. Non-Cryst. Solids, 378 (2013) 273-281. 
[32] E.M. Pierce, P. Frugier, L.J. Criscenti, K.D. Kwon, S.N. Kerisit, Int. J. Appl. Glass Sci., in press (2014).

[33] P. Frugier, C. Martin, I. Ribet, T. Advocat, S. Gin, J. Nucl. Mater., 346 (2005) 194-207.

[34] E.M. Pierce, E.L. Richards, A.M. Davis, L.R. Reed, E.A. Rodriguez, Environ. Chem., 5 (2008) 73-85.

[35] A. Ledieu, F. Devreux, P. Barboux, L. Sicard, O. Spalla, J. Non-Cryst. Solids, 343 (2004) 312.

[36] C. Jegou, S. Gin, F. Larche, J. Nucl. Mater., 280 (2000) 216-229.

[37] S. Gin, X. Beaudoux, F. Angéli, C. Jégou, N. Godon, J. Non-Cryst. Solids, 358 (2012) $2559-2570$.

[38] P. Frugier, S. Gin, Y. Minet, T. Chave, B. Bonin, N. Godon, J.-E. Lartigue, P. Jollivet, A. Ayral, L. De Windt, G. Santarini, J. Nucl. Mater., 380 (2008) 8-21.

[39] P. Frugier, T. Chave, S. Gin, J.-E. Lartigue, J. Nucl. Mater., 392 (2009) 552-567.

[40] F. Delage, D. Ghaleb, J.L. Dussosoy, O. Chevalier, E. Vernaz, J. Nucl. Mater., 190 (1992) 191-197.

[41] L.-H. Kieu, J.M. Delaye, L. Cormier, C. Stolz, J. Non-Cryst. Solids, 357 (2011) 3313-3321. 